\title{
Fusion of Infrared and Visible Images Based on Pulse Coupled Neural Network and Nonsubsampled Contourlet Transform
}

\author{
Song Jianhui, Gan Jing and Liu Yanju*
}

\author{
School of Information Science and Engineering, Shenyang Ligong University, Shenyang, Liaoning, 110159, China
}

\begin{abstract}
This paper presents a new fusion algorithm which can effectively solve the problem that has unobvious infrared target and low contrast in infrared and visible image fusion. This paper's innovation point is its fusion rule. Other algorithms' fusion rules usually use pulse coupled neural network (PCNN) and region characteristics to select low frequency or bandpass subband coefficients. The proposed algorithm innovatively applies improved PCNN and region characteristics to the selection of both low frequency and bandpass subband coefficients in nonsubsampled contourlet transform (NSCT) domain. First, the subband coefficients of original image are obtained by NSCT. Then, the decomposed subband coefficients are processed by using PCNN, whose fire mapping images are obtained. The method of region standard deviation is used to choose the fusion coefficients of fire mapping image, which acquires more image information in low frequency part. For bandpass subband coefficients' fire mapping image, the method based on region energy is adopted for fusion coefficients, which makes the bandpass part capture more energy. Finally, the fused image can be obtained by inverse transform of NSCT. Compared with typical wavelet-based, NSCT-based and NSCT-PCNN based fusion algorithms, experiment shows that the new proposed algorithm can improve the fused image's objective evaluation index significantly, obtaining a prominent infrared target and better fusion image quality.
\end{abstract}

Keywords: Image fusion, Infrared and visible, PCNN, NSCT, Region characteristics.

\section{INTRODUCTION}

Image fusion is a process in which multiple sensor images are processed to extract useful information of each image, fusing a new image. It combines the advantages of each sensor image, contrasting with the same scene, with its representations being richer than a single sensor image [1]. Currently, domestic and foreign scholars are conducting an indepth research on infrared and visible images fusion. Infrared image has interference ability and low resolution. Visible image is rich in spectral information, but is easily affected by the external factors. The two types of images exist complementary in the objective, which compensates their shortcomings by integration, to give full play to their favorable properties and to get more fully accurate image information. Fusion of infrared and visible images in practical applications is very broad, especially in military reconnaissance, target recognition, medical image, security surveillance and remote sensing.

There are pyramid transform algorithm [2-4], wavelet transform algorithm [5, 6], curvelet transform algorithm [7] and contourlet transform algorithm [8] for infrared and visible image fusion. But these algorithms have some deficiencies. The nonsubsampled contourlet transform (NSCT) [9] is an excellent image multi-scale analysis tool. It has timefrequency localization characteristic, anisotropy and shiftinvariance. Through NSCT, sparse representation of image can be obtained. It has received widespread attention for

*Address correspondence to this author at the No. 6, Middle Nanping Road, Hunnan District, Shenyang City, Liaoning Province, China. Postcard: 110159; Tel: 15840583885; E-mail: 20080571@qq.com fusion of infrared and visible images. Liu Guixi et al. [3] adopted contrast pyramid method to decompose the infrared and visible image, using the weighted average method in the low frequency part, and to apply the selection and weighted average method based on rectangle region characteristic measurement in the high frequency part except the top layer. In the process, the fused images have good visual effect through selectively highlighting the comparison with different frequency bands of the image. Liu Kun et al. [10] segmented the infrared and visible image considering the spatial frequency as the target area measurement in NSCT domain. Detecting the edge region of infrared and visible image, combined with the visible image's back ground region, fusion strategy was proposed, at last obtaining the fused image. It not only could effectively capture the infrared target, but also maintain the background information of visible image. Guo et al. [11] adopted the strategy to mix similar structure and energy distribution of the energy area together to obtain the image fusion. Li Meili et al. [12] adopted edge method in low frequency subband coefficients of NSCT domain, and used the improved pulse coupled neural network (PCNN) to choose fusion coefficients in the high frequency part, and adopted PCNN's [13] global coupling and pulse synchronization of neurons to obtain better fusion effect. A variety of methods have been proposed for infrared and visible image fusion, but the most appropriate method has not been found yet.

In image fusion, the fusion rules play an important role. More extensive use of fusion rule involves the pixel, such as taking the maximum absolute values of the fusion coefficients, combining selection and weighted average which 


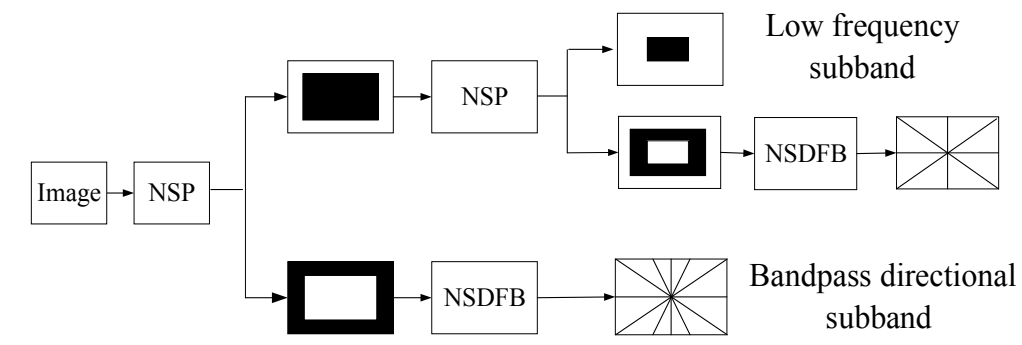

Fig. (1). Schematic diagram of NSCT.

have been proposed by Burt and others [14]. Details of the features in the image are usually expressed by pixels in the same region, and the pixels in the same region have a close relationship with each other [5]. Fusion rules based on region make the energy, standard deviation and spatial frequency the characteristic operators, which process all the pixels in a region, and are more ideal compared with the image fusion based on simple pixel fusion rules.

In order to obtain more outstanding infrared target and higher contrast fused image, combining NSCT, PCNN and region characteristics, the paper proposed a new fusion rule. In the NSCT domain, the PCNN and region characteristics are applied innovatively to low and high frequency coefficients selection. The fusion rule is: first, the image is decomposed by NSCT, and in the NSCT domain, the improved PCNN is used to process the low frequency and high frequency subband image and fire mapping maps in low and high frequency are obtained. The selection of fusion coefficients of low frequency fire mapping maps is made on the basis of taking maximum absolute value method and the combination of region standard deviation. The fusion coefficient in high frequency fire mapping maps adopts the method of taking maximum absolute value and combining the region energy. Finally, through subjective and objective comparison among the proposed algorithm and other algorithms, the validity of the proposed algorithm can be proven.

\section{FUSION RULES AND FUSION STEPS}

\subsection{NSCT}

The nonsubsampled contourlet transform (NSCT) consists of two parts: nonsampled pyramid (NSP) and nonsubsampled directional filter banks (NSDFB). It only uses upsample filter, gets the shift-in variance, overcomes the frequency aliasing and eliminates the fusion image of pseudoGibbs phenomenon. The NSCT schematic diagram is shown in Fig. (1).

Firstly, the image is decomposed by NSP and low frequency subband image and bandpass subband image are obtained with the same size as the source image. Then, the band pass subband images in multiple directions are captured by NSDFB. According to the number of the image decompositions, the low frequency subband decomposition above is repeated until it is completed. In this paper, the infrared and visible image decomposition is carried out by NSCT, which obtains coefficients $T_{I, L}(m, n), T_{V, \mathrm{~L}}(m, n)$ of low frequency subband, and bandpass subband coefficients $T K I, J(m, n)$ and $T K V, J(m, n)$ in the NSCT domain. $J$ is the decomposition layer and $k$ is the direction number. With the proposed fusion rules to select the decomposed subband coefficients, the final image fusion coefficients $B_{F, L}(m, n)$ and $H K F, J(m, n)$ are obtained, and by reconstructing the final fusion coefficients in the NSCT domain, the fusion image can be achieved.

\subsection{Fusion Rule Based on PCNN and Region Standard Deviation for Low Frequency Part}

The simplified and improved version of Eckhornneuron model [15] is shown in Fig. (2). This model consists of three parts: the input area, the input connection region and the pulse generator. The input area receives the external incentive, transmitting signal to the input connection through the channels $F$ and $L$. Due to interaction with the pulse generator, the output $Y$ is finally obtained.

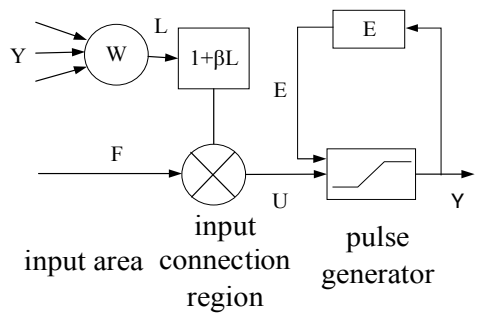

Fig. (2). Improved PCNN model.

The iterative formula of the improved model is as follows:

$$
\begin{aligned}
& F_{i j}(n)=I_{i j} \\
& L_{i j}(n)=e^{-\alpha_{L}} L_{i j}(n-1)+V_{L} \sum_{k l} W_{i j k l} Y_{k l}(n-1) \\
& U_{i j}(n)=F_{i j}(n)\left(1+\beta L_{i j}(n)\right) \\
& Y_{i j}(n)=\left\{\begin{array}{l}
1, U_{i j}(n)>E_{i j}(n-1) \\
0, H_{\text {其他 }}
\end{array}\right. \\
& E_{i j}(n)=e^{-\alpha_{E}} E_{i j}(n-1)+V_{E} Y_{i j}(n-1)
\end{aligned}
$$

$I_{i j}$ is the external incentive for neurons pixel gray value, $L_{i j}$ is the connection of the input neurons, $U_{i j}$ is the internal network, $Y_{i}$ is the output of network, $E_{i j}$ is the dynamic threshold and $n$ is the maximum number of iterations. $B$ is the connection strength. $V_{L}$ and $V_{E}$ are amplification coefficients of dynamic internal neural network connected with domain $L$ and threshold $E$. Their decay time constants are respectively $\alpha_{L}$ and $\alpha_{E}$. $W_{i j k l}$ refers to the connecting range neurons for the coupled domain. The connection range neurons are $k$ and $l$. The specific process of the network is that neurons receive external incentives of channel $F$ and other 
neurons input from the channel $L . U_{i j}$ is attained through formula (3) with internal activities. If $U_{i j}$ is bigger than the dynamic threshold that is, $E, Y_{i j}(n)=1$, it means that the neurons are ignited, and if $Y_{i j}(n)=0$, it means that they are not ignited. When neurons are ignited, the dynamic threshold suddenly increases and the pulse output stops. When the threshold is reduced to less than $U_{i j}$, the neuron is ignited again and produces ignition pulse output, which is repeated until the maximum number of iterations is reached.

In the image processing of PCNN, the number of neurons in the network is the same as the number of image pixels to be processed, with each pixel having a corresponding neuron. Pixel gray values are taken as input neurons of the external motivation and the pulse output is obtained through internal neurons processing. In the maximum number of iterations, ignition output of all the neurons is acquired. The output of neural image ignition is the fire mapping images by PCNN processing.

Low frequency component of the image contains the general characteristics of image, such as the whole contour, the contrast of the image, the target information etc., which is the approximate expression of an image. Therefore, the quality of low frequency part of the image fusion plays a very important role. In this algorithm, the gray level of low frequency and high frequency coefficients in NSCT domain are considered as the value of PCNN external excitation input. Connection range set of PCNN neurons is set to 5 and the maximum number of iterations is set to 200 . For the processing of low frequency part, the method of taking max absolute value is used combined with region standard deviation. Region standard deviation expresses the discrete case of region pixel gray value, the whole average gray pixel and the image data. The greater the standard deviation and the more obvious the difference among the pixels, and the more image information the region gets. In addition, the greater the absolute value is and the greater the pixel value is, the greater the information is obtained. Firstly, large absolute values of coefficients in the low frequency part of fire mapping images are obtained, so that most of the energy of the image can be acquired. When the absolute values of the low frequency part in the two images are equal, larger region standard deviation is adopted, which is considered as the fusion coefficient. The fusion rules are shown as follows: $D_{I, L}(m, n), D_{V, L}(m, n)$ are the low frequency coefficients of infrared and visible images in the fire mapping image; $\sigma_{I, L}(m, n), \sigma_{V, L}(m, n)$ are the standard deviations of infrared and visible images in the fire mapping image. The region window is set to $3 \times 3$ and $B_{F, L}(m, n)$ is set as low frequency fusion coefficient.

$B_{F, L}(m, n)=\left\{\begin{array}{l}D_{I, L}(m, n), a b s\left[D_{V, L}(m, n)\right]<a b s\left[D_{I, L}(m, n)\right] \\ D_{V, L}(m, n), a b s\left[D_{V, L}(m, n)\right]>a b s\left[D_{I, L}(m, n)\right]\end{array}\right.$

When the absolute values $D_{I, L}(m, n)$ and $D_{V, L}(m, n)$ are equal, fusion coefficient in low frequency is shown as follows:

$B_{F, L}(m, n)=\left\{\begin{array}{l}D_{I, L}(m, n), \sigma_{V, L}(m, n) \leq \sigma_{I, L}(m, n) \\ D_{V, L}(m, n), \sigma_{V, L}(m, n)>\sigma_{I, L}(m, n)\end{array}\right.$

\subsection{Fusion Rule Based on PCNN and Region Energy for the High Frequency Part}

The high frequency part of the image represents the image edge information, which is crucial to the clarity of the image, so that the reasonable treatment can make the fused images more clear. The more the pixel ignition times of maps of the high frequency subband, the more abundant the image information and the better visual effect are achieved. But in processing of the ignition times on the single pixel, the pixel's correlation is ignored, which loses the detailed information of the local information. This in turn influences the fusion effect, so the paper made use of the region energy fusion rule. Through the region energy comparison of ignition map in high frequency, fusion coefficient was selected, and the iteration number of simulation was set to 200. Fusion rules are shown as follows:

(1) The gray value of high frequency coefficient in NSCT domain is considered an external PCNN input, through iterative calculation, getting the fire mapping images. High frequency coefficients of visible and infrared images after progressing are $C K(m, n)$, and $C K I, J(m, n)$, in which, $J$ is the NSCT decomposition layer and $K$ represents each layer in the direction number.

(2) Comparing the absolute value of high frequency coefficient, the larger value is obtained. When the absolute values are equal, their region energies are compared and the higher ones are chosen.

(3) The region energy of the high frequency coefficients is calculated whose area of the window is set to $3 \times 3$; $E K$ $I, J(m, n), E K V,(m, n)$ are region energies of infrared and visible images. The calculation formula is shown as follows:

$$
\begin{aligned}
& E_{I, J}^{K}(m, n)=\sum_{j=0}^{2} \sum_{i=0}^{2} \boldsymbol{w}(i+2, j+2)\left[C_{I, J}^{K}(m+i, n+j)\right]^{2} \\
& E_{V, J}^{K}(m, n)=\sum_{j=0}^{2} \sum_{i=0}^{2} \boldsymbol{w}(i+2, j+2)\left[C_{V, J}^{K}(m+i, n+j)\right]^{2}
\end{aligned}
$$

$w=\left[\begin{array}{lllllll}0.6 & 0.8 & 0.6 ; 0.8 & 1 & 0.9 ; 0.6 & 0.8 & 0.6\end{array}\right], w$ is the window mask matrix, whose center weight is 1 . The closer the matrix elements to the center, the greater the weight is.

(4) The high frequency fusion formula is as follows:

$$
\begin{aligned}
& H_{F, J}^{\mathrm{K}}(m, n)= \\
& \left\{\begin{array}{l}
C_{I, J}^{K}(m, n), a b s\left[C_{V, J}^{K}(m, n)\right]<a b s\left[C_{I, J}^{K}(m, n)\right] \\
C_{V, J}^{K}(m, n), a b s\left[C_{V, J}^{K}(m, n)\right]>a b s\left[C_{I, J}^{K}(m, n)\right]
\end{array}\right.
\end{aligned}
$$

When absolute values of the high frequency coefficients are equal, comparing the region energy, the greater value is selected as the fusion coefficient.

$$
\begin{aligned}
& H_{F, J}^{\mathrm{K}}(m, n)= \\
& \left\{\begin{array}{l}
C_{I, J}^{K}(m, n), E_{V, J}^{K}(m, n)<E_{I, J}^{K}(m, n) \\
C_{V, J}^{K}(m, n), E_{V, J}^{K}(m, n) \geq E_{I, J}^{K}(m, n)
\end{array}\right.
\end{aligned}
$$




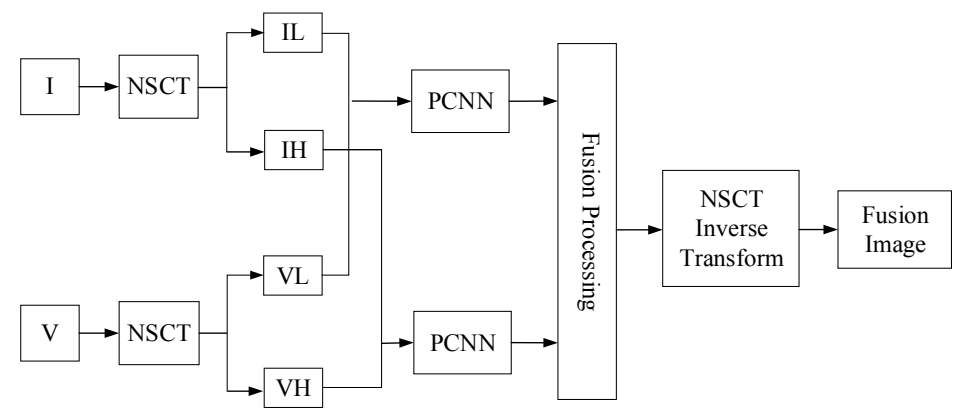

Fig. (3). The realization of image fusion algorithm.

\subsection{Image Fusion Steps}

Infrared and visible images have a strict registration before fusion. Fig. (3) describes the algorithm realization.

(1) The visible image $V$ and the infrared image $I$ are decomposed by NSCT and the low and high frequency coefficients of each image are achieved.

(2) The gray value of low and high frequency coefficients of NSCT domain is considered as the external input. Fire mapping images are obtained in low and high frequencies by the iterative processing of PCNN. According to the formula (6) (7), the low frequency fusion coefficients $B_{\mathrm{F}, \mathrm{L}}(m, n)$ are obtained by the method based on the absolute value and the region standard deviation.

(3) According to the formula (8) (11), using the method of absolute value and region energy, high frequency fusion coefficients $H \mathrm{~K}$ F,L $(m, n)$ are achieved.

(4) Low and high frequency fusion coefficients $B_{\mathrm{F}, \mathrm{L}}(m, n)$ and $H \mathrm{~K} F, \mathrm{~L}(m, n)$ are reconstructed respectively by NSCT, to obtain the fused image.

\section{RESULTS AND DISCUSSION}

The paper compared and analyzed wavelet-based, NSCTbased, NSCT-PCNN-based and innovation algorithms in image fusion. In the experiment of wavelet-based algorithm, "Haar" was chosen as the wavelet basis, the fusion rules obtained average low frequency coefficients, taking the max absolute value of high frequency coefficients. The fusion rules of NSCT-based algorithm were the same as of the wavelet-based. The fusion rules of NSCT-PCNN-based algorithm adopted average low frequency coefficients, and it was the same with the innovation algorithm in high frequency part. All the directional filters of NSCT used "9-7", multiscale filters adopted "pkva", and decomposition layer of the four algorithms was set to 4 .

Fig. (4) shows the original images and the fusion images of the above algorithms. Fig. (4a) represents the original visible image, and 4(b) shows the original infrared image. Fig. (4c) represents the fused image of wavelet-based algorithm, Fig. (4d) shows the fused image of NSCT-based algorithm, Fig. (4e) displays the fused image of NSCT-PCNNbased algorithm and Fig. (4f) shows the fused image of innovation algorithm.

In Fig. (4), background information of the visible image and target of the infrared image can be extracted by all of the four algorithms. In Fig. (4c), block effect is obvious, edge detail information is fuzzy, contrast is not high and infrared target is not outstanding enough. Fig. (4d) is better than (4c) in terms of the clarity, which is better for distinguishing the details of background information, but it has poor brightness than (4c), and the edge information of infrared target and background part is not smooth enough. The effect of Fig. (4e) in the infrared target is highlighted than Figs. (4c) and (4d), but is not obvious; however, it is much clearer in terms of image details in contrast with Figs. (4c) and (4d). But the effect of Fig. (4e) is poor compared to Fig. (4f). The effect shown in Fig. (4f) is superior in terms of brightness, contrast and clarity than Figs. (4c-4e), and its infrared target characteristic is obvious, edge profile is clear and visual effect is the best.

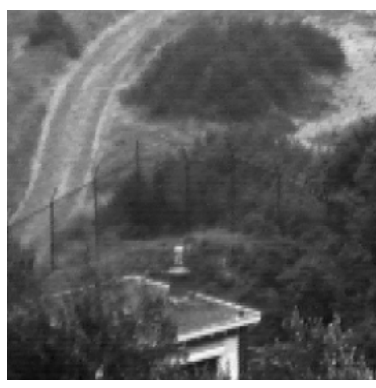

4(a)

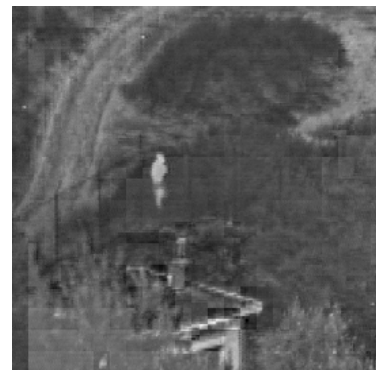

4(c)

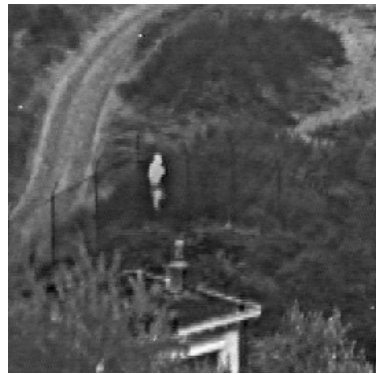

$4(e)$

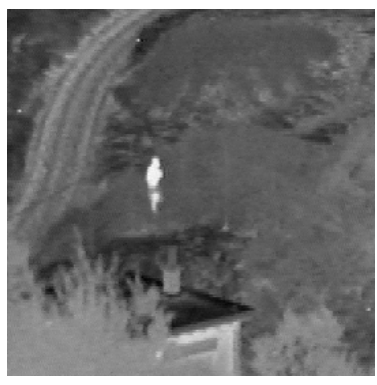

4(b)

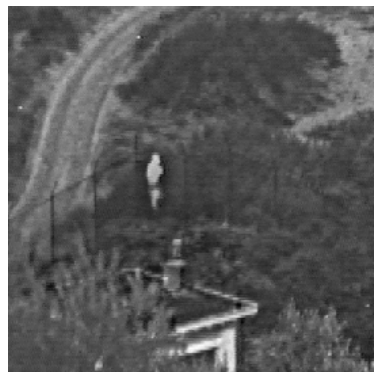

4(d)

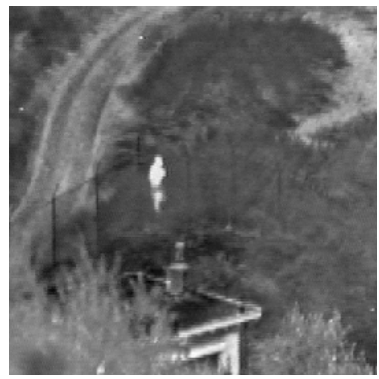

4(f)
Fig. (4). The contrast of the original images and fused images of the four algorithms. 
Table 1. Objective evaluation of the fused images.

\begin{tabular}{|c|c|c|c|c|}
\hline & Wavelet & NSCT & NSCT- PCNN & Innovation Algorithm \\
\hline \hline Entropy & 6.5228 & 6.6051 & 6.6729 & 6.9179 \\
\hline Mean & 90.2618 & 87.8552 & 81.7531 & 99.3177 \\
\hline Standard Deviation & 28.6917 & 31.6453 & 33.5261 & 34.3939 \\
\hline Clarity & 1.6865 & 1.9477 & 2.0607 & 2.0893 \\
\hline Mutual Information & 1.4186 & 1.3331 & 1.3697 & 1.6405 \\
\hline Transfer Entropy & 0.5507 & 0.5047 & 0.5163 & 0.5406 \\
\hline
\end{tabular}

Table 1 presents the objective evaluation of the original image and the various algorithms' fused images, adopting entropy, mean, transfer entropy, standard deviation, clarity and mutual information. Entropy is used to measure the abundance degree of image information. The larger the entropy, the more information the image contains. The mean represents the average of gray image pixel values to characterize the overall brightness of the image. Image clarity is an important criterion. The more defined the image, the more obvious the details. Standard deviation can measure the dispersion of the gray image. The larger the image disperses, the higher the visual performance is in contrast. Mutual information measures how much information the fused image captures from the original image. The greater the mutual information, the more the image information is captured. Transfer entropy indicates the amount of information transfer between the original image and the fused image. Higher values indicate more information transfers, and better quality of the fused image.

According to objective evaluation, shown from the data in Table 1, mean, mutual information and transfer entropy of NSCT method were less than the wavelet transform method. But the entropy, standard deviation and clarity were better than the wavelet transform method. The mean of NSCTPCNN was smaller than NSCT, but the rest of the indexes were better than the NSCT method. The transfer entropy of innovation algorithm was less than the wavelet transform, but the rest of the indexes were optimal in the four kinds of algorithms. Compared with the wavelet-based algorithm, the entropy increased by $6.06 \%$, the mean increased by $10.03 \%$, standard deviation increased by $19.87 \%$, clarity increased by $23.88 \%$ and mutual information increased by $15.64 \%$. Compared with the NSCT-based algorithm, the entropy increased by $4.74 \%$, the mean increased by $13.05 \%$, the standard deviation increased by $8.69 \%$, the clarity increased by $7.27 \%$, the mutual information increased by $23.06 \%$ and the transfer entropy increased by $7.11 \%$. Compared with the NSCTPCNN-based algorithm, the entropy increased by $3.67 \%$, the mean had an increase by $21.48 \%$ while the standard deviation increased by $2.59 \%$. The clarity increased by $1.39 \%$, the mutual information increased by $19.77 \%$ and the transfer entropy also increased by $4.71 \%$. Through comparison data, in the condition of the same fusion rules, the image details which were captured by NSCT-based algorithm were more than the wavelet-based algorithm, and the fusion image was much clearer. The difference between NSCT-PCNN-based algorithm and NSCT-based algorithm was based on the fu- sion rules in the high frequency part of the image. The objective evaluation index was better than the NSCT-based algorithm, and the innovation algorithm in this study appeared superior to the NSCT-PCNN-based algorithm. It can be concluded that the validity of the proposed fusion rules and new fusion algorithm is superior to the other three algorithms.

\section{CONCLUSION}

In this paper, the unique advantages of PCNN in the visual aspects and of NSCT in the image sparse representation were combined. The proposed fusion rule based on PCNN and region characteristics in NSCT domain was put forward to solve the problem that infrared target was less obvious and image contrast was low in visible and infrared image fusion. Through a variety of experiments, the paper obtained the fused images of innovation algorithm, wavelet-based algorithm, NSCT-based algorithm and NSCT-PCNN-based algorithm. On the basis of subjective analysis and objective evaluation, the results show that the new algorithm is better than the above three algorithms, the infrared target of fusion images is prominent, the contrast is high and the edge details are clear. This demonstrates the validity and superiority of this new algorithm.

\section{CONFLICT OF INTEREST}

The authors confirm that this article content has no conflict of interest.

\section{ACKNOWLEDGEMENTS}

The study is funded by the 2014 Education Department of Liaoning Province (Project No.: L2014079). The authors would like to thank Dr. Lex Toet of TNO Human Factors Research Institute for providing the images used in this paper. The insightful comments and suggestions of the anonymous reviewers obviously contributed to the improvement of this paper.

\section{REFERENCES}

[1] D.L. Hall, and J. Llinas, "An introduction to multi sensor data fusion", In: Proceedings of the IEEE, vol. 85, no.1, pp. 6-23, Jan. 1997.

[2] A. Toet, "Hierarchical image fusion", Machine Vision and Applications, vol. 3, no. 1, pp.1-11, 1990.

[3] G. X. Liu, S. G. Zhao, and W. J. Chen, "Multi-resolution scheme appropriate to fusing infrared and visible light images", Journal of Optoelectronics Laser, (in Chinese), vol. 15, no.8, pp. 980-984, 2004. 
[4] P. J. Burt, and E.H. Adelson, "The laplacian pyramid as a compact Image code", IEEE Transactions on Communications, vol. 31, no. 4, pp. 532-540, Apr., 1983.

[5] G. X. Liu, and W. H. Yang, "A wavelet-decomposition-based image fusion scheme and its performance evaluation", Acta Automatica Sinica, vol. 28, no. 6, pp. 927-934, 2002.

[6] G Pajares, and J. M. de la Cruz, "A wavelet-based image fusion tutorial”, Pattern Recognition, vol. 37, no. 9, pp. 1855-1872, 2004.

[7] E. Candes, L. Demanet, D. Donoho, L. Ying, "Fast discrete curvelet transforms", Multiscale Modeling and Simulation, vol. 5, no. 3, pp. 861-899, 2006.

[8] M.N. Do, and M. Vetterli, "The contourlet transform: an efficient directional multi resolution image representation", IEEE Transactions on Image Processing, vol. 14, no. 12, pp. 2091-2106, Dec. 2005

[9] A. L. D. Cunha, J. Zhou, M. N. Do, "The Nonsubsampled Contourlet Transform: Theory, Design, and Applications", IEEE Transactions on Image Processing, vol. 15, no.10, pp. 3089-3101, Oct. 2006
[10] K. Liu, L. Guo, H. H. Li, J. Chen, "Fusion of infrared and visible light images based on region segmentation", Chinese Journal of Aeronautics, vol. 22, no. 1, pp. 75-80, 2009.

[11] B. Guo, Q. Zhang, Y. Hou, "Region-based fusion of infrared and visible images using nonsubsampled contourlet transform", Chinese Optics Letters, vol. 6, no. 5, pp. 338-341, 2008.

[12] M. L. Li, Y. J. Li, H. M. Wang, K. Zhang, "Fusion algorithm of infrared and visible images based on NSCT and PCNN", OptoElectronic Engineering, vol. 37, no. 6, pp. 90-95, 2010. (in Chinese)

[13] R. Eckhorn, H. J. Reitboeck, M. Arndt, P. Dicke, "Feature linking via synchronization among distributed assemblies: Simulations of results from cat visual cortex", Neural Computation (S0899-7667), vol. 2, no. 3, pp. 293-307, 1990.

[14] R. Chao, K. Zhang, and Y. J. Li, "An image fusion algorithm using wavelet transform", Acta Electronica Sinica, vol. 32, no. 5, pp. 750-753, 2004.

[15] Y. W. Bi, and T. S. Qiu "An adaptive image segmentation method based on a simplified PCNN", Acta Electronica Sinica, vol. 33, no. 4, pp. 647-650, 2005.

Received: September 22, 2014

Revised: November 30, 2014

Accepted: December 02, 2014

(C) Jianhui et al.; Licensee Bentham Open.

This is an open access article licensed under the terms of the Creative Commons Attribution Non-Commercial License (http://creativecommons.org/licenses/by-nc/4.0/) which permits unrestricted, non-commercial use, distribution and reproduction in any medium, provided the work is properly cited. 